\title{
A TOY MODEL FOR GAMMA-RAY BURSTS IN TYPE IB/C SUPERNOVAE
}

\author{
WEI-HUA LEI, DING-XIONG WANG ${ }^{1}$, AND REN-YI MA \\ Department of Physics, Huazhong University of Science and Technology, Wuhan, 430074, People's \\ Republic of China \\ dxwang@hust . edu.cn
}

\begin{abstract}
A toy model for gamma-ray burst-supernovae (GRB-SN) is discussed by considering the coexistence of baryon poor outflows from black holes $(\mathrm{BHs})$ and a powerful spin-connection to surrounding disk, giving rise to consistent calorimetry as described by van Putten (2001a) in a variant of the Blandford-Znajek process (BZ, 1977). In this model the half-opening angle of the magnetic flux tube on the horizon is determined by the mapping relation between the angular coordinate on the $\mathrm{BH}$ horizon and the radial coordinate on the surrounding accretion disk. The GRB is powered by the baryon poor outflows in the BZ process, and the associated $\mathrm{SN}$ is powered by very small fraction of the spin energy transferred from the BH to the disk in the magnetic coupling (MC) process. The timescale of the GRB is fitted by the duration of the open magnetic flux on the horizon. It turns out that the data of several GRB-SNe are well fitted with our model.
\end{abstract}

Subject headings: black hole physics-accretion disks-gamma-ray bursts-supernovae

\section{INTRODUCTION}

As is well known, the Blandford-Znajek (BZ) process is an effective mechanism for powering jets from quasars and active galactic nuclei (Blandford \& Znajek 1977; Rees 1984). In the BZ process energy and angular momentum are extracted from a rotating balck hole $(\mathrm{BH})$, and transferred to the remote astrophysics load by the open magnetic field lines. Recently, much attention has been paid on the issue of the long duration gamma-ray bursts (GRBs) powered by the BZ process (Paczynski 1993, 1998; Meszaros \& Rees 1997). A detailed model of GRBs invoking the BZ process is given by Lee, Wijers, \& Brown 2000, hereafter L00), and they concluded that a fast-spinning BH with strong magnetic field $\sim 10^{15} \mathrm{G}$ can provide energy $\sim 10^{53} \mathrm{ergs}$ to power a GRB within 1000 seconds.

Recently the observations and theoretical considerations have linked long duration GRB with ultrabright type Ib/c supernovae (Galama et al. 1998; Bloom et al. 1999; Galama et al. 2000). The first candidate was provided by SN1998bw and GRB980425, and the recent HETE-II burst GRB030329 has greatly enhanced the confidence in this association (Stanek et al. 2003; Hjorth et al. 2003).

\footnotetext{
${ }^{1}$ Send offprint requests to: Ding-Xiong Wang (dxwang@hust.edu.cn)
} 
Not long ago Brown et al. (2000, hereafter B00) worked out a specific scenario for GRB-SN connection. They argued that the GRB is powered by the BZ process, and the SN is powered by the energy dissipated into the disk through closed magnetic field lines coupling the disk with the BH. The latter energy mechanism is referred to as the magnetic coupling (MC) process, which is regarded as one of the variants of the BZ process (Blandford 1999; Putten 1999; Li 2000, 2002; Wang, Xiao \& Lei 2002, hereafter W02). It is shown in B00 that about $10^{53}$ ergs are available to power both GRBs and SNe. However, they failed to distinguish the fractions of the energy for these two objects.

More recently, van Putten et al. (van Putten 2001a; van Putten \& Levinson 2003; van Putten et al. 2004) worked out a poloidal topology for the open and closed magnetic field lines, where the separatrix on the horizon is defined by a finite half-opening angle. The duration of GRB is set by the lifetime of rapid spin of the BH. It is found that GRB and SN are powered by a small fraction of the $\mathrm{BH}$ spin energy. This result is consistent with the observations, i.e., the duration of GRB with tens of seconds, the true GRB energies distributed around $5 \times 10^{50}$ ergs (Frail et al. 2002), and the aspherical SNe kinetic energies with $2 \times 10^{51}$ ergs ( Hoflich et al. 1999).

In this paper we propose a toy model for GRB-SN by considering coexistence of the BZ and MC processes (CEBZMC), where the configuration of the magnetic field is based the works of Wang et al. and van Putten et al. (W02; Wang et al. 2003, hereafter W03; van Putten 2001a; van Putten et al. 2004). The transferred energy and the duration for GRBs are calculated in the evolving process of the half-opening angle of the magnetic flux on the BH horizon, while the transferred energy for SNe is calculated in the MC process. Thus the association of GRBs and SNe is explained reasonably and consistently.

This paper is organized as follows. In $\S 2$ the configuration of the magnetic field is described with the half-opening angle $\theta_{B Z}$ of the magnetic flux tube on the horizon, and we find that $\theta_{B Z}$ can evolve to zero in some range of the power-law index $n$ of the magnetic field on the disk. In $\S 3$ the toy model for GRB-SN based on BH evolution is described in the parameter space consisting of the BH spin $a_{*}$ and the power-law index $n$. It is shown that the half-opening angle $\theta_{B Z}$ plays an important role of determining (i) the powers for GRBs and SNe, (ii) the duration of GRB. In $\S 4$ we calculate the duration of GRB and the energies of GRB-SN, and compare with the other models of GRBs invoking the BZ process. It is found the duration of GRB obtained in our model is shorter than that of the other models. It turns out that this model is in excellent agreement with the observations, owing to considering the MC effects, while too much energy than the needed energy of GRB is produced in the other models without the $\mathrm{MC}$ effects. Finally, in $\S 5$, we summarize our main results and discuss some problems concerning our model. Throughout this paper, the geometric units $G=c=1$ are used.

\section{CONFIGURATION OF THE MAGNETIC FIELD}

Recently, we proposed a model of CEBZMC, in which the remote astrophysical load in the BZ process and the disk load in the MC process are connected with a rotating $\mathrm{BH}$ by open and closed magnetic field lines, respectively (W02; W03). The poloidal configuration of the magnetic field is shown in Figure 1, which is adapted from van Putten (2001a).

In Figure 1 the angle $\theta_{B Z}$ is the half-opening angle of the open magnetic flux tube, indicating the angular boundary between open and closed field lines on the horizon. This angle has been discussed by van Putten and his collaborators, who related the half-opening angle to the curvature in poloidal 
topology of the inner torus magnetosphere (van Putten \& Levinson 2003; van Putten et al. 2004). In W03 the angle $\theta_{B Z}$ is determined based on the following assumptions.

(1) The theory of a stationary, axisymmetric magnetosphere formulated in the work of MacDonald \& Thorne (1982) is applicable not only to the BZ process but also to the MC process. The magnetosphere is assumed to be force-free outside the $\mathrm{BH}$ and disk.

(2) The disk is both stable and perfectly conducting, and the closed magnetic field lines are frozen in the disk. The disk is thin and Keplerian, and it lies in the equatorial plane of the $\mathrm{BH}$, with inner boundary being at the marginally stable orbit.

(3) The poloidal magnetic field is assumed to be constant on the horizon and to vary as a power law on the disk as follows (Blandford 1976),

$$
B_{D} \propto \xi^{-n}
$$

where $B_{D}$ is the magnetic field on the disk, the parameter $n$ is the power-law index, and $\xi \equiv r / r_{m s}$ is the radial coordinate on the disk, which is defined in terms of the radius $r_{m s} \equiv M \chi_{m s}^{2}$ of the marginally stable orbit (Novikov \& Thorne 1973).

(4) The magnetic flux connecting the $\mathrm{BH}$ with its surrounding disk takes precedence over that connecting the $\mathrm{BH}$ with the remote load.

Assumption (4) is proposed based on two reasons: (i) the magnetic field on the horizon is brought and held by the surrounding magnetized disk, and (ii) the disk is much nearer to the $\mathrm{BH}$ than the remote load.

A mapping relation between the parameter $\xi$ and the angular $\theta$ is derived in W03 based on the conservation of magnetic flux of the closed field lines.

$$
\cos \theta=\int_{1}^{\xi} \mathrm{G}\left(a_{*} ; \xi, n\right) d \xi
$$

where $a_{*} \equiv J / M^{2}$ is the $\mathrm{BH}$ spin defined by the BH mass $M$ and angular momentum $J$, and the function $G\left(a_{*} ; \xi, n\right)$ is given by

$$
\mathrm{G}\left(a_{*} ; \xi, n\right)=\frac{\xi^{1-n} \chi_{m s}^{2} \sqrt{1+a_{*}^{2} \chi_{m s}^{-4} \xi^{-2}+2 a_{*}^{2} \chi_{m s}^{-6} \xi^{-3}}}{2 \sqrt{\left(1+a_{*}^{2} \chi_{m s}^{-4}+2 a_{*}^{2} \chi_{m s}^{-6}\right)\left(1-2 \chi_{m s}^{-2} \xi^{-1}+a_{*}^{2} \chi_{m s}^{-4} \xi^{-2}\right)}} .
$$

Based on the assumption (4) the angle $\theta_{B Z}$ can be determined by taking $\xi=\infty$ for the highest closed field lines as follows,

$$
\cos \theta_{B Z}=\int_{1}^{\infty} \mathrm{G}\left(a_{*} ; \xi, n\right) d \xi
$$

The curves of $\theta_{B Z}$ versus $a_{*}$ with different values of power-law index $n$ are shown in Figure 2.

Inspecting Figure 2, we have the following results. 
(1) The half-opening angle $\theta_{B Z}$ always increases monotonically with the increasing $n$.

(2) For some values of $n$, such as $n=3.5,4,4.5,5$ and 5.493, the angle $\theta_{B Z}$ can evolve to zero with the decreasing $a_{*}$.

The second point implies that the open magnetic flux tube will be shut off when the BH spin decreases to the critical value $a_{*}^{G R B}$ corresponding to $\theta_{B Z}=0$. The lifetime of the half-opening angle $\theta_{B Z}$ is defined as the evolution time of $\mathrm{BH}$ from the initial spin $a_{*}(0)$ to $a_{*}^{G R B}$. Obviously it is less than the lifetime of $\mathrm{BH}$ spin, which is the evolution time of BH from $a_{*}(0)$ to zero. By using equation (4) we find that $a_{*}^{G R B}$ exists only for a specific value range of the power-law index $n$, i.e., $3.003 \leq n \leq 5.493$. The half-opening angle $\theta_{B Z}$ remains zero for $n<3.003$ in the whole evolving process, and it will never evolve to zero for $n>5.493$.

\section{A TOY MODEL FOR GRB-SN BASED ON BH EVOLUTION IN CEBZMC}

Considering that the angular momentum is transferred from a rapidly rotating $\mathrm{BH}$ to the disk, on which a positive torque exerts, we think the accretion onto the $\mathrm{BH}$ might be probably halted. This state is essentially the suspended accretion state proposed by van Putten \& Ostriker (2001), and the evolution of the $\mathrm{BH}$ is governed by the $\mathrm{BZ}$ and the $\mathrm{MC}$ processes.

The expressions for the BZ and MC powers and torques are derived in W02 by using an improved equivalent circuit for the BH magnetosphere based on the work of MacDonald and Thorne (1982). Considering the angular boundary $\theta_{B Z}$, we express the BZ and $\mathrm{MC}$ powers and torques as follows.

$$
\begin{gathered}
\tilde{P}_{B Z} \equiv P_{B Z} / P_{0}=2 a_{*}^{2} \int_{0}^{\theta_{B Z}} \frac{k(1-k) \sin ^{3} \theta d \theta}{2-(1-q) \sin ^{2} \theta}, \\
\tilde{T}_{B Z} \equiv T_{B Z} / T_{0}=4 a_{*}(1+q) \int_{0}^{\theta_{B Z}} \frac{(1-k) \sin ^{3} \theta d \theta}{2-(1-q) \sin ^{2} \theta}, \\
\tilde{P}_{M C} \equiv P_{M C} / P_{0}=2 a_{*}^{2} \int_{\theta_{B Z}}^{\pi / 2} \frac{\beta(1-\beta) \sin ^{3} \theta d \theta}{2-(1-q) \sin ^{2} \theta}, \\
\tilde{T}_{M C} \equiv T_{M C} / T_{0}=4 a_{*}(1+q) \int_{\theta_{B Z}}^{\pi / 2} \frac{(1-\beta) \sin ^{3} \theta d \theta}{2-(1-q) \sin ^{2} \theta},
\end{gathered}
$$

where we have $q \equiv \sqrt{1-a_{*}^{2}}$ and

$$
\left\{\begin{array}{l}
P_{0} \equiv\left\langle B_{H}^{2}\right\rangle M^{2} \approx 6.59 \times 10^{50} \times\left(\frac{B_{H}}{10^{15} G}\right)^{2}\left(\frac{M}{M_{\odot}}\right)^{2} \mathrm{erg} \cdot \mathrm{s}^{-1} \\
T_{0} \equiv\left\langle B_{H}^{2}\right\rangle M^{3} \approx 3.26 \times 10^{45}\left(\frac{B_{H}}{10^{15 G}}\right)^{2}\left(\frac{M}{M_{\odot}}\right)^{3} \mathrm{~g} \cdot \mathrm{cm}^{2} \cdot \mathrm{s}^{-2}
\end{array}\right.
$$

In equation (9) $B_{H}$ is the magnetic field on the $\mathrm{BH}$ horizon. The parameters $k$ and $\beta$ are the ratios of the angular velocities of the open and closed magnetic field lines to that of the $\mathrm{BH}$, respectively. 
Usually, $k=0.5$ is taken for the optimal BZ power. Since the closed field lines are assumed to be frozen in the disk, the ratio $\beta$ is related to $a_{*}$ and $\xi$ by

$$
\beta \equiv \Omega_{D} / \Omega_{H}=\frac{2(1+q)}{a_{*}\left[\left(\sqrt{\xi} \chi_{m s}\right)^{3}+a_{*}\right]},
$$

where $\Omega_{H}$ and $\Omega_{D}$ are the angular velocities of the $\mathrm{BH}$ and the disk, respectively.

Since $\theta_{B Z}$ in equations (5) and (7) is determined by $a_{*}$ and $n$, we have the curves of the powers $\tilde{P}_{B Z}$ and $\tilde{P}_{M C}$ versus $a_{*}$ for the given values of $n$ as shown in Figure 3.

Inspecting Figure 3, we have the following results.

(1) The MC power $P_{M C}$ is always greater than the BZ power $P_{B Z}$ for a wide value range of the power-law index $n$, provided that the BH spin $a_{*}$ is not very small.

(2) For the given values of $a_{*}$ the BZ power $P_{B Z}$ increases with the increasing $n$, while the MC power $P_{M C}$ decreases with it.

(3) The power $P_{M C}=0$ holds at some small values of $a_{*}$ for the given values of the index $n$.

From equation (5) we find $P_{B Z}=0$ when $\theta_{B Z}=0$. Therefore the effective time of the BZ process can be set by the lifetime of $\theta_{B Z}$, i.e., the evolution time of $\mathrm{BH}$ from $a_{*}(0)$ to $a_{*}^{G R B}$ for $3.003 \leq n \leq 5.493$. As argued in our previous work (Wang, Lei \& Ma 2003), we have $P_{M C}=0$ when the energy transported from the $\mathrm{BH}$ to the disk is equal to that from the disk to the $\mathrm{BH}$. Henceforth the corresponding critical $\mathrm{BH}$ spin is denoted by $a_{*}^{S N}$. We have $P_{M C}>0$ for $a_{*}>a_{*}^{S N}$, and $P_{M C}<0$ for $a_{*}<a_{*}^{S N}$, indicating that the energy transferred from the $\mathrm{BH}$ to the disk dominates that transferred in the inverse direction.

Based on the conservation laws of energy and angular momentum we have the following evolution equations of the rotating $\mathrm{BH}$,

$$
\begin{gathered}
d M / d t=-\left(P_{B Z}+P_{M C}\right), \\
d J / d t=-\left(T_{B Z}+T_{M C}\right) .
\end{gathered}
$$

Incorporating equations (11) and (12), we have the evolution equation for the BH spin expressed by

$$
d a_{*} / d t=-M^{-2}\left(T_{B Z}+T_{M C}\right)+2 M^{-1} a_{*}\left(P_{B Z}+P_{M C}\right)=B_{H}^{2} M A\left(a_{*}, n\right)
$$

where the function $A\left(a_{*}, n\right)$ is

$$
A\left(a_{*}, n\right)=2 a_{*} \tilde{P}_{m a g}-\tilde{T}_{m a g}
$$

where 


$$
\tilde{P}_{m a g}=\tilde{P}_{B Z}+\tilde{P}_{M C}, \tilde{T}_{m a g}=\tilde{T}_{B Z}+\tilde{T}_{M C}
$$

The sign of $A\left(a_{*}, n\right)$ determines whether $a_{*}$ decreases or increases, and we have the curves of $A\left(a_{*}, n\right)$ versus $a_{*}$ for different values of $n$ as shown in Figure 4.

From Figure 4, we find the following evolution characteristics of the BH spin.

(1) The function $A\left(a_{*}, n\right)>0$ for $a_{*}<a_{*}^{e q}$, and $A\left(a_{*}, n\right)<0$ for $a_{*}>a_{*}^{e q}$, and the BH spin always evolve to the equilibrium spin $a_{*}^{e q}$.

(2) Both the equilibrium spin $a_{*}^{e q}$ and the decreasing rate of the BH spin are sensitive to the powerlaw index $n$. The greater the index $n$ is, the greater is the equilibrium spin $a_{*}^{e q}$, and the more slowly the $\mathrm{BH}$ spin decreases to $a_{*}^{e q}$.

(3) The features of our model for GRB-SN can be described by three functions: $A\left(a_{*}, n\right), \tilde{P}_{B Z}\left(a_{*}, n\right)$ and $\tilde{P}_{M C}\left(a_{*}, n\right)$.

Setting $A\left(a_{*}, n\right)=0, \tilde{P}_{B Z}\left(a_{*}, n\right)=0$ and $\tilde{P}_{M C}\left(a_{*}, n\right)=0$, we have the three characteristic curves in the parameter space as shown in Figure 5.

The parameter space is divided into seven regions, IA, IB, II, IIIA, IIIB, IVA and IVB, where the points $\boldsymbol{M}$ and $\boldsymbol{N}$ are the intersections of the curve $\tilde{P}_{B Z}\left(a_{*}, n\right)=0$ with the curves $\tilde{P}_{M C}\left(a_{*}, n\right)=0$ and $A\left(a_{*}, n\right)=0$, respectively. IA and IB is divided by the dashed line starting from the point $\boldsymbol{M}$. By using the equations (5), (7) and (14) we obtain the coordinates of points $\boldsymbol{M}$ and $\boldsymbol{N}$, i.e., $\boldsymbol{M}(\mathbf{0 . 2 5 3}$, 5.067) and $\boldsymbol{N}(\mathbf{0 . 2 2 2}, \mathbf{5 . 1 2 2})$, respectively. In Figure 5 each black dot with an arrowhead in these sub-regions is referred to as a representative point $(\mathbf{R P})$, which represents one evolution state of the $\mathrm{BH}$ as shown in Table 1.

It is found from Figure 5 and Table 1 that GRB-SN only occurs in region $\mathbf{I}$ and $\mathbf{I}$ corresponding to CEBZMC. Based on the observations that GRBs need less energy than SNe (B00), we think that the evolving path of the $\mathbf{R P}$ in region IA is reasonable, which corresponds to $3.003<n<5.067$, and the RP attains $\tilde{P}_{B Z}\left(a_{*}, n\right)=0$ with $\tilde{P}_{M C}\left(a_{*}, n\right)>0$ in braking the spinning $\mathrm{BH}$.

Based on the above discussion we describe the toy model for GRB-SN in the parameter space as follows.

(1) The fast-spinning BH can power GRBs and SNe via the BZ and MC processes, respectively, which is represented by the $\mathbf{R P}$ in region IA. The GRB will be shut off, when the $\mathbf{R P}$ reaches eventually the curve $\tilde{P}_{B Z}\left(a_{*}, n\right)=0$.

(2) Energy is transferred from the spinning BH to the disk via the MC process in the evolving path from region IA to region II. The SN will be shut off with no further energy transferred into the disk, if the $\mathbf{R P}$ arrives at the curve $\tilde{P}_{M C}\left(a_{*}, n\right)=0$.

(3) Since enormous energy is deposited in the disk due to the MC process, the disk might be destroyed eventually, leaving the $\mathrm{BH}$ alone without the disk. This outcome probably arises from explosion of $\mathrm{SNe}(\mathrm{B} 00)$. 


\section{ENERGIES AND TIME SCALES FOR GRB-SN}

In the evolving path of the $\mathbf{R P}$ going through regions IA, II and IIIA we have $a_{*}^{G R B} \geq a_{*}^{S N}>a_{*}^{e q}$. Based on the above discussion on correlation of the BH evolution with the association of GRB-SN, the energy $E_{B Z}$ and $E_{S N}$ extracted in the BZ and MC processes are given respectively by

$$
\begin{gathered}
E_{B Z}=\int_{a_{*}(0)}^{a_{*}^{G R B}} \frac{P_{B Z}}{\left(d a_{*} / d t\right)} d a_{*}=1.79 \times 10^{54} \mathrm{ergs} \times\left(\frac{M(0)}{M_{\odot}}\right) \int_{a_{*}(0)}^{a_{*}^{G R B}} \frac{\tilde{M} \tilde{P}_{B Z}}{-\tilde{T}_{\text {mag }}+2 a_{*} \tilde{P}_{\text {mag }}} d a_{*}, \\
E_{M C}=\int_{a_{*}(0)}^{a_{*}^{S N}} \frac{P_{M C}}{\left(d a_{*} / d t\right)} d a_{*}=1.79 \times 10^{54} \mathrm{ergs} \times\left(\frac{M(0)}{M_{\odot}}\right) \int_{a_{*}(0)}^{a_{*}^{S N}} \frac{\tilde{M} \tilde{P}_{M C}}{-\tilde{T}_{m a g}+2 a_{*} \tilde{P}_{m a g}} d a_{*} .
\end{gathered}
$$

The true energy for GRBs, $E_{\gamma}$, and the energy for SNe, $E_{S N}$, are related respectively to $E_{B Z}$ and $E_{M C}$ by

$$
\begin{gathered}
E_{\gamma}=\varepsilon_{\gamma} E_{B Z}, \\
E_{S N}=\varepsilon_{S N} E_{M C},
\end{gathered}
$$

where $\varepsilon_{\gamma}$ and $\varepsilon_{S N}$ denote the efficiencies of converting $E_{B Z}$ and $E_{M C}$ into $E_{\gamma}$ and $E_{S N}$, respectively. Following van Putten et al. (2004), we take $\varepsilon_{\gamma}=0.15$ in calculations. The duration of GRB, $t_{G R B}$, is defined as the lifetime of the half-opening angle $\theta_{B Z}$, which is exactly equal to the time for the BH spin evolving from $a_{*}(0)$ to $a_{*}^{G R B}$, i.e.,

$$
t_{G R B}=2.7 \times 10^{3} s \times\left(\frac{10^{15} G}{B_{H}}\right)^{2}\left(\frac{M_{\odot}}{M(0)}\right) \int_{a_{*}(0)}^{a_{*}^{G R B}} \frac{\tilde{M}^{-1}}{-\tilde{T}_{m a g}+2 a_{*} \tilde{P}_{m a g}} d a_{*},
$$

where $\tilde{M}$ is the ratio of the BH mass to its initial value $M(0)$, and it is calculated by

$$
\tilde{M} \equiv \frac{M}{M(0)}=\exp \int_{a_{*}(0)}^{a_{*}} \frac{d a_{*}}{\tilde{T}_{m a g} / \tilde{P}_{m a g}-2 a_{*}} .
$$

Equation (21) can be derived by using equations (11) and (13). Obviously, $t_{G R B}$ is shorter than the lifetime of the $\mathrm{BH}$ spin evolving from $a_{*}(0)$ to $a_{*}=0$.

Considering that spin-energy of the BHs produced in core-collapse is around $50 \%$ of maximum or less in centered nucleation (van Putten 2004), we take the initial BH spin as $a_{*}(0)=0.9$ in equations (16), (17) and (21). The value range $3.534<n<5.067$ is taken in the evolving path of the $\mathbf{R P}$ in region IA corresponding to GRB-SN. In addition, $M(0)=7 M_{\odot}$ and $B_{H}=10^{15} G$ are assumed, and the cutoff of $T_{G R B}$ is taken as $T_{90}$ in calculations, which is the duration for $90 \%$ of the total BZ energy to be extracted (Lee \& Kim 2002). 
As is well know, the rotational energy $E_{\text {rot }}$ of a Kerr BH is expressed by (Thorne, Price \& Macdonald 1986)

$$
E_{\text {rot }}=f\left(a_{*}\right) M(0)
$$

where

$$
f\left(a_{*}\right)=1-\sqrt{\frac{1}{2}\left(1+\sqrt{1-a_{*}^{2}}\right)}
$$

Substituting $a_{*}(0)=0.9$ and $M(0)=7 M_{\odot}$ into equation $(22)$, we have the total rotational energy of the $\mathrm{BH}$,

$$
E_{\text {rot }} \approx 1.9 \times 10^{54} \text { ergs }
$$

To compare rotational energy of the $\mathrm{BH}$ extracted in the $\mathrm{BZ}$ and $\mathrm{MC}$ processes, we calculate the ratios of $E_{B Z}$ and $E_{M C}$ to $E_{r o t}$, i.e.,

$$
\eta_{B Z} \equiv E_{B Z} / E_{r o t}, \quad \eta_{M C} \equiv E_{M C} / E_{r o t}
$$

The curves of $\eta_{B Z}$ and $\eta_{M C}$ versus $n$ are shown in Figure 6.

From Figure 6 we find that $E_{M C}$ is significantly greater than $E_{B Z}$, and the greater the indexn, the more energy extracted by BZ process. These results can be also obtained by inspecting Figures 3 and 5 . The ratio $\eta_{M C}$ varies from $25.8 \%$ to $33.8 \%$ with the ratio $\eta_{B Z}$ varying from zero to $3.7 \%$ for $3.534<n<5.067$. Combining equations $(24)$ and (25) with the above ratios, we obtain $E_{M C}$ varying from $4.929 \times 10^{53} \mathrm{ergs}$ to $6.459 \times 10^{53} \mathrm{ergs}$ with $E_{B Z}=7.003 \times 10^{52} \mathrm{ergs}$. And the energy for GRB is inferred by using $\varepsilon_{\gamma}=0.15$.

Calculations of SN1998bw with aspherical geometry show that the needed kinetic energy is about $2 \times 10^{51} \mathrm{ergs}$ (Hoflich et al. 1999), and this results in the ratio $\varepsilon_{S N}$ varying from 0.003 to 0.004 for $E_{M C}$ varying from $6.459 \times 10^{53} \mathrm{ergs}$ to $4.929 \times 10^{53} \mathrm{ergs}$ in equation (19). In this paper we take the average value, $\varepsilon_{S N} \approx 0.0035$.

It has been shown that most fraction of the rotational energy of a Kerr BH is emitted in unseen channels, such as in gravitational radiation and MeV-neutrino emissions (van Putten 2001a, 2001b; van Putten and Levinson 2002). The above efficiency factors obtained in our model, $\varepsilon_{\gamma}=0.15$ and $\varepsilon_{S N} \approx 0.0035$, imply that only very small fraction of $E_{r o t}$ is converted into the energy for GRBs and SNe. These results are consistent with those obtained by van Putten and his collaborators.

By using equation (20) the duration of GRBs, $T_{90}$, versus the parameter $n$ is shown in Figure 7.

We find that the estimated duration of GRB is tens of seconds, which is consistent with the observations (Kouveliotous et al. 1993). For $n=4$ and $B_{H}=1 \times 10^{15} G$ in the evolving path of the RP from $a_{*}(0)=0.9$ to $a_{*}^{S N}=0.235$, we obtain the duration of GRB $T_{90}=21 s$, and the energies for GRB and $\mathrm{SN}$ are $E_{\gamma}=5 \times 10^{50} \operatorname{ergs}\left(\frac{\varepsilon_{\gamma}}{0.15}\right)$ and $E_{S N}=2 \times 10^{51} \operatorname{ergs}\left(\frac{\varepsilon_{S N}}{0.0035}\right)$, respectively. These results are in excellent agreement with the observed durations of tens of seconds (Kouveliotous et al. 1993), energies 
$E_{\gamma}=5 \times 10^{50}$ ergs in gamma rays (Frail et al. 2002), and inferred kinetic energy $E_{S N}=2 \times 10^{51}$ ergs in SN1998bw with aspherical geometry (Hoflich et al. 1999).

For several GRB-SNe, the observed energy $E_{\gamma}$ and duration $T_{90}$ can be fitted by adjusting the parameters $n$ and $B_{H}$, and the energy $E_{S N}$ can be predicted as shown in Table 2.

In the previous model for GRBs powered by the BZ process, the duration of GRB is estimated either in the case that the $\mathrm{BH}$ is spun down to zero or in the case that the whole disk is plunged into the BH (L00; Lee \& Kim 2000, 2002; Wang, Lei \& Xiao 2002). However, the MC effects have not been taken into account in these models. In this model the duration of GRB is estimated by the lifetime of the half-opening angle on the BH horizon based on CEBZMC. It turns out that both the BZ and MC processes play very important roles for GRN-SN in this model.

The quantities, $E_{\gamma}$ and $T_{90}$, calculated in the three different models for GRBs are listed in Table 3, where the abbreviations CEBZMC, BZO and BZACC represent this model, the model invoking the BZ process only, the model invoking the BZ process with disk accretion, respectively.

It is found in Table 3 that the energy and duration time obtained in this model are in excellent agreement with the observed durations of tens of seconds and energies $E_{\gamma} \approx 5 \times 10^{50} \mathrm{ergs}$. However the energies obtained in the models BZO and BZACC seem too much to fit the observations.

\section{DISCUSSION}

In this paper we discuss a toy model for GRB-SN, where the energy for GRBs and SNe are powered by the $\mathrm{BZ}$ and $\mathrm{MC}$ processes, respectively, and the duration for GRB is estimated by the lifetime of the half-opening angle $\theta_{B Z}$. The energy extracted in the BZ process for GRBs is much less than that extracted in the MC process for SNe. This result is consistent with the observations: the true energy of GRB $E_{\gamma}=5 \times 10^{50} \mathrm{ergs}$ and the aspherical SNe of kinetic energy $E_{S N}=2 \times 10^{51} \mathrm{ergs}$. For a set of GRBs the observed true energy $E_{\gamma}$ and duration $T_{90}$ can be well fitted by taking adequate values of the power-law index $n$ and magnetic field $B_{H}$. However, there are still several issues related to this model.

(i) In this paper we take the evolving path of the $\mathbf{R P}$ in the region IA to describe the association of GRB-SN as shown in Figure 5. In this case the GRB will terminate before the SN event stops. In fact the evolving path in region IB can be also used to describe the association of GRB-SN, and it implies that the $\mathbf{R P}$ will arrive at the characteristic curve of $\tilde{P}_{M C}=0$ ahead of reaching the characteristic curve of $\tilde{P}_{B Z}=0$. However, we think that the GRB will still terminate before the SN event stops, because the disk will be destroyed during the explosion of the $\mathrm{SN}$, and the BZ process can not work without the magnetic field supported by its surrounding disk.

(ii) In order to highlight the effects of the BZ and MC processes in powering GRB-SN, we neglect the effects of the disk accretion in this model. In fact, disk accretion plays a very important role in $\mathrm{BH}$ evolution. The slowing down of the $\mathrm{BH}$ spin will be delayed significantly since the accreting matter delivers remarkable amount of energy and angular momentum to the BH. Strictly speaking, disk accretion cannot be halted steadily by the MC process. The MC effects on disk accretion is a very complicated, and it should be treated dynamically.

(iii) According to Kruskal-Shafranov criteria (Kadomtsev 1966), the screw instability will occur if the toroidal magnetic field becomes so strong that the magnetic field lines turns around itself about once. 
In our latest work (Wang et al. 2004), we argued that the state of CEBZMC always accompanies the screw instability. In this model the half-opening angle $\theta_{B Z}$ is related to the infinite radial parameter $\xi$ by equation (4). We expect to derive a greater $\theta_{B Z}$ by considering the restriction of the screw instability to the $\mathrm{MC}$ region. Thus the ratios $\eta_{G R B}$ and $\eta_{S N}$ will be changed. We shall improve this model in future.

\section{Acknowledgments}

We thank the anonymous referees for numerous constructive suggestions. This work is supported by the National Natural Science Foundation of China under grants 10173004, 10373006 and 10121503.

\section{REFERENCES}

Blandford, R. D. 1976, MNRAS, 176, 465

Blandford, R. D. 1999, in ASP Conf. Ser. 160, Astrophysical Discs: An EC Summer School, ed. J. A. Sellwood \& J. Goodman (San Francisco: ASP), 265

Blandford, R. D., \& Znajek, R. L. 1977, MNRAS, 179, 433

Bloom, J. S. et al. 1999, Nature, 401, 453

Brown, G. E., et al. 2000, New Astronomy 5, 191 (B00)

Costa, E., et al. 1997, IAUC Circ. No. 6649

Dar, A. 2004, astro-ph/0405386

Frail, D. A., et al. 2002, ApJ, 562, L55

Galama, T. J., et al. 1998, Nature, 395, 672

Galama, T. J., et al. 2000, ApJ, 536, 185

Heise, J., et al. 1999, IAUC Circ. No. 7221

Hjorth, J., et al. 2003, Nature, 423, 847

Hoflich, P. J., Wheeler, J. C., \& Wang, L. 1999, ApJ, 521, 179

Kadomtsev, B. B. 1966, Rev. Plasma Phys., 2, 153

Kouveliotou et al. 1993, Astrophys. J. Lett., 413, L101

Lee, H. K., \& Wijers, R. A. M. J., Brown, G. E. 2000, Phys. Rep., 325, 83 (L00)

Lee, H. K., \& Kim, H. K. 2000, J. Korean Phys. Soc. 36, 188

Lee, H. K., \& Kim, H. K. 2002, J. Korean Phys. Soc. 40, 524 
Li, L. -X. 2000, ApJ, 533, L115

Li, L. -X. 2002, ApJ, 567, 463

MacDonald, D., \& Thorne, K. S. 1982, MNRAS, 198, 345

Meszaros P., \& Rees M. J. 1997, ApJ, 482, L29

Novikov, I. D., \& Thorne, K. S. 1973, in Black Holes, ed. Dewitt C (Gordon and Breach, New York) p. 345

Paczynski, B. 1993, in Relativistic Astrophysics and Particle Cosmology, ed. C. W. Akerlof \& M. A. Srednicki (Ann. NY Acad. Sci., Vol. 688), 321

Paczynski, B. 1998, ApJ, 494, L45

Rees, M. J. 1984, ARA\&A. 22, 471

Stanek, K. Z. et al. 2003, ApJ, 591, L17

Thorne, K. S., Price, R. H., \& Macdonald, D. A. 1986, Black Holes: The Membrane Paradigm, Yale Univ. Press, New Haven

van Putten, M. H. P. M., 1999, Science, 284, 115

van Putten, M. H. P. M. 2001a, Phys. Rep., 345, 1

van Putten, M. H. P. M. 2001b, Phys. Rev. Lett., 87, 091101

van Putten, M. H. P. M., \& E. C. Ostriker, E. C. 2001, ApJ, 552, L31

van Putten, M. H. P. M., Levinson, A. 2002, Science, 295, 1874

van Putten, M. H. P. M., Levinson, A. 2003, ApJ, 584, 937

van Putten, M. H. P. M., Levinson, A., Regimbau, T., Punturo, M., \& Harry, G. M. 2004, Phys. Rev. $\mathrm{D}, 69,044007$

van Putten, M. H. P. M. 2004, astro-ph/0407112 (to appear in ApJ Lett.)

Wang, D.-X., Lei, W.-H., \& Xiao, K. 2002, ApJ, 580, 358

Wang, D.-X., Xiao, K., \& Lei, W.-H. 2002, MNRAS, 335, 655 (W02)

Wang, D.-X., Ma, R.-Y., Lei, W.-H., \& Yao, G.-Z. 2003, ApJ, 595, 109 (W03)

Wang, D.-X., Lei W.-H., \& Ma, R.-Y. 2003, MNRAS, 342, 851

Wang, D.-X., Ma, R.-Y., Lei, W.-H., \& Yao, G.-Z. 2004, ApJ, 601, 1031 
Table 1. Characteristic of $\mathrm{BH}$ evolution in each region of the parameter space

\begin{tabular}{cccccc}
\hline \hline Region & $d a_{*} / d t$ & $P_{B Z}$ & $P_{M C}$ & RP displacement & Possible events \\
\hline IA & $<0$ & $>0$ & $>0$ & Towards the left & GRB-SN \\
IB & $<0$ & $>0$ & $>0$ & Towards the left & GRB-SN \\
II & $<0$ & - & $>0$ & Towards the left & SN \\
IIIA & $<0$ & - & $<0$ & Towards the left & $\overline{\text { GRB }}$ \\
IIIB & $<0$ & $>0$ & $<0$ & Towards the left & $\overline{ }$ \\
IVA & $>0$ & - & $<0$ & Towards the right & $\overline{\text { GRB }}$ \\
IVB & $>0$ & $>0$ & $<0$ & Towards the right & ( \\
\hline
\end{tabular}

Table 2. Four GRBs of the given true energy $E_{\gamma}$ and $T_{90}$ s fitted with the different power-law index $n$ and the predicted $E_{S N}$

\begin{tabular}{cccccc}
\hline \hline GRB $^{\mathrm{a}}$ & $E_{\gamma}\left(10^{51}\right.$ ergs $^{\mathrm{b}}$ & $T_{90}(\mathrm{~s})$ & $n$ & $B_{H}\left(10^{15} G\right)$ & $E_{S N}{ }^{\mathrm{c}}$ \\
\hline 970508 & 0.234 & $15^{\mathrm{d}}$ & 3.885 & 0.97 & $1.947 \times 10^{51} \operatorname{ergs}\left(\frac{\varepsilon_{S N}}{0 \hat{\varepsilon}_{S N}^{0035}}\right)$ \\
990712 & 0.445 & $30^{\mathrm{e}}$ & 3.975 & 0.81 & $1.989 \times 10^{51} \operatorname{ergs}\left(\frac{0.9035}{0.0035}\right)$ \\
991208 & 0.455 & $39.84^{\mathrm{f}}$ & 3.985 & 0.75 & $1.995 \times 10^{51} \operatorname{ergs}\left(\frac{\dot{\varepsilon}_{S N}^{0.35}}{0 \dot{\varepsilon}_{S N}^{0035}}\right)$ \\
991216 & 0.695 & $7.51^{\mathrm{f}}$ & 4.058 & 1.80 & $2.032 \times 10^{51} \operatorname{ergs}\left(\frac{0.0035}{0.0035}\right)$ \\
\hline
\end{tabular}

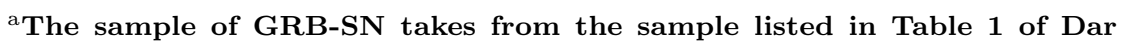
(2004)

${ }^{\mathrm{b}}$ The true energy $E_{\gamma}$ of GRB refers to the sample listed in Table 1 of Frail et al (2002)

${ }^{\mathrm{c}}$ The predicted energy of SNe based on our model

${ }^{\mathrm{d}}$ The duration of GRB970508 refers to Costa et al (1997)

eThe duration of GRB990712 refers to Heise et al. (1999)

${ }^{\mathrm{f}}$ The durations of GRB991208 and GRB991216 refer to the sample listed in Table 2 of Lee \& Kim (2002)

Table 3. Energy and duration obtained in three models for GRBs with $a_{*}(0)=0.9, M_{H}(0)=7 M_{\odot}$, $B_{H}=10^{15} G$ and the initial disk mass $M_{D}(0)=3 M_{\odot}$

\begin{tabular}{ccc}
\hline \hline Model & $E_{\gamma}($ ergs $)$ & $T_{90}(\mathrm{~s})$ \\
\hline BZO & $1.225 \times 10^{53}$ & 348 \\
BZACC & $1.734 \times 10^{53}$ & 397 \\
CEBZMC & $<1.050 \times 10^{52}$ & $<115$ \\
\hline
\end{tabular}




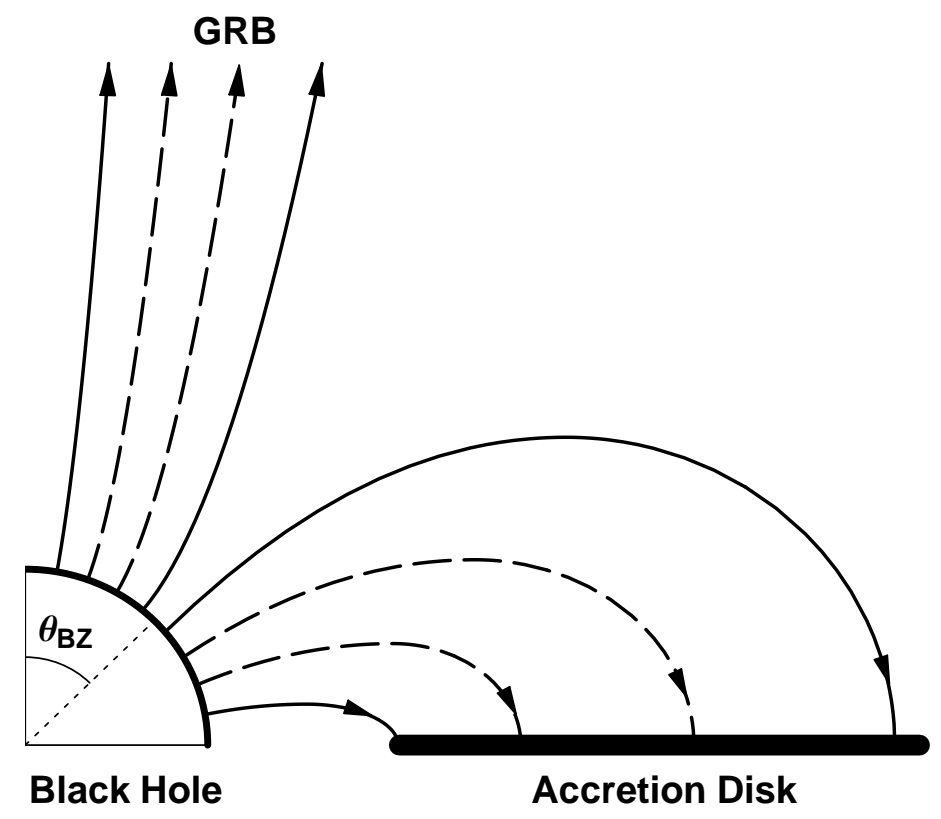

Fig. 1. - Poloidal magnetic field connecting a rotating $\mathrm{BH}$ with remote astrophysical load and a surrounding disk 


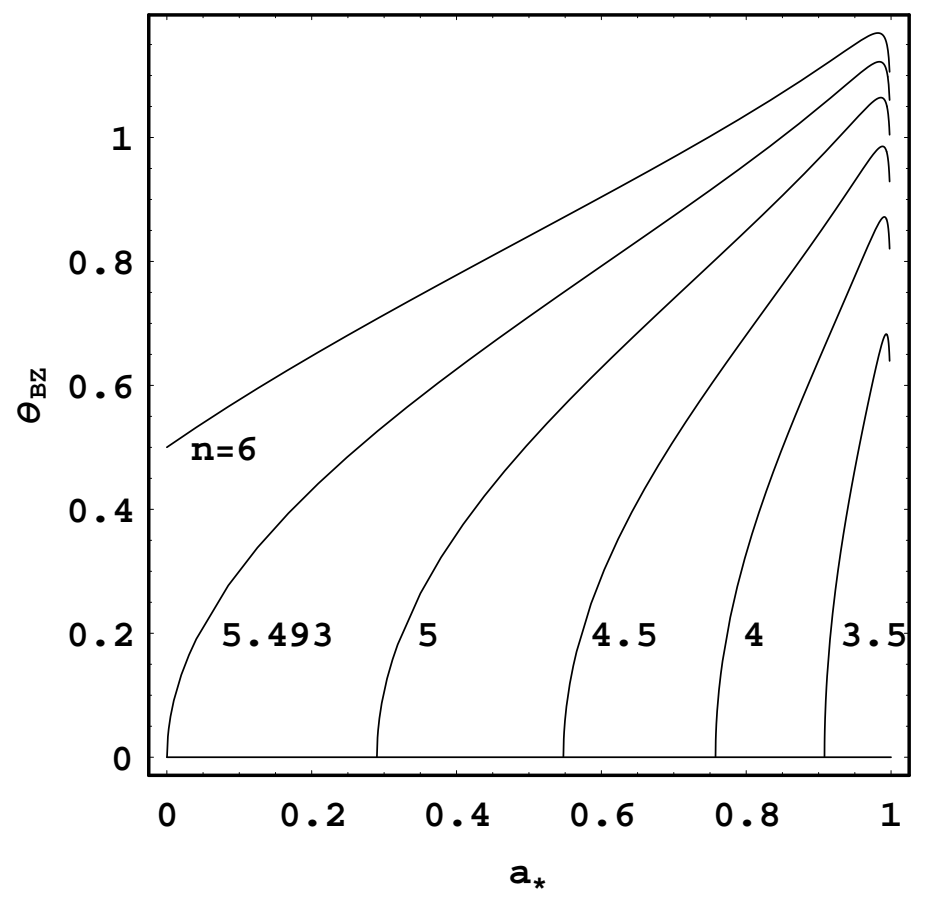

Fig. 2.- Curves of $\theta_{B Z}$ vs. $a_{*}$ for $n=3.5,4,4.5,5,5.493$ and 6 . 


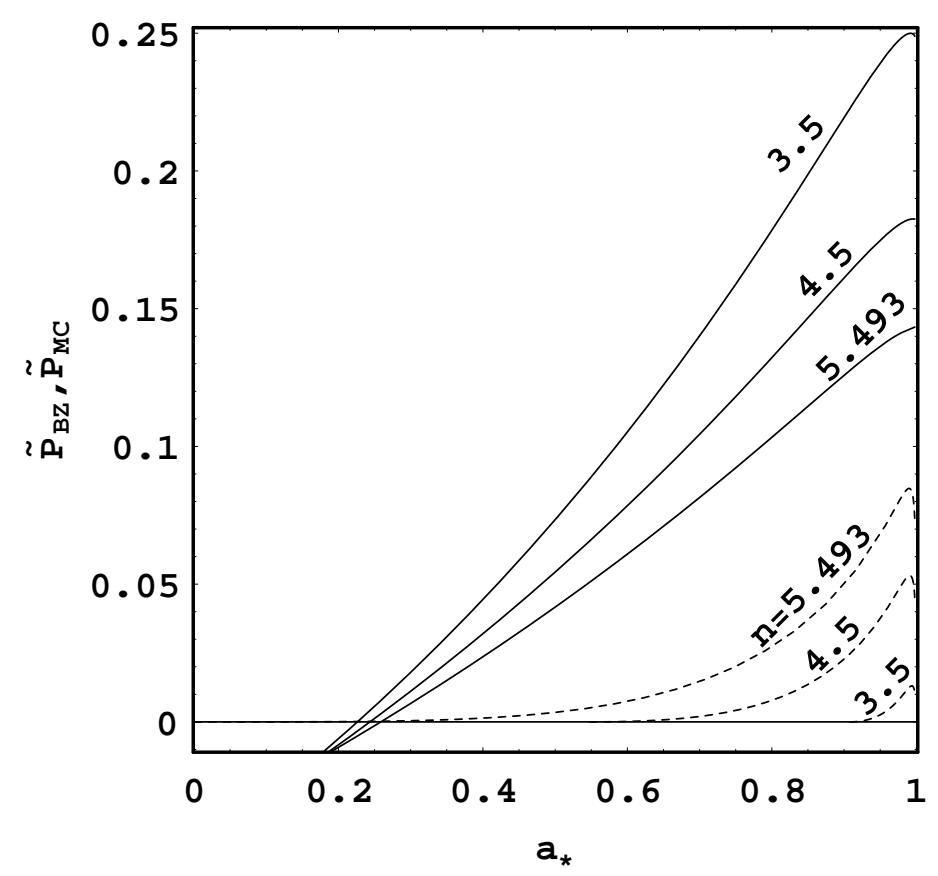

Fig. 3.- Curves of $\tilde{P}_{B Z}$ (dotted lines) and $\tilde{P}_{M C}$ (solid lines) vs. $a_{*}$ with $n=3.5,4.5$ and 5.493 for $0<a_{*}<1$. 


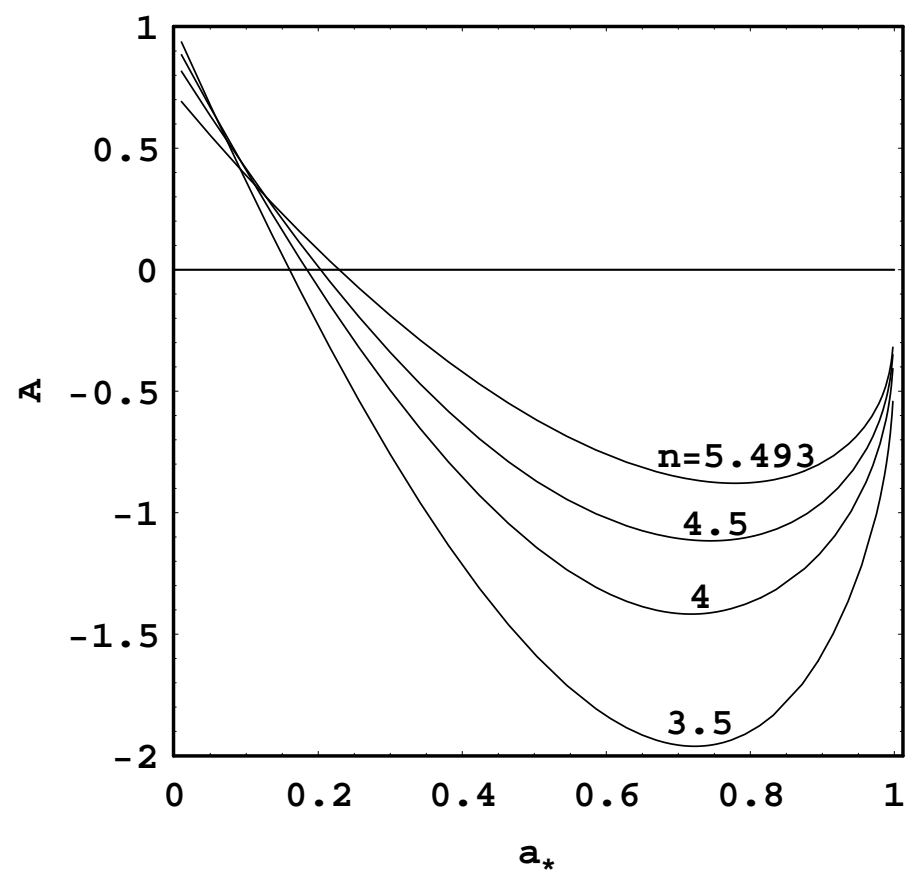

Fig. 4.- Curves of $A\left(a_{*}, n\right)$ vs. $a_{*}$ for $n=3.5,4,4.5$ and 5.493 . 


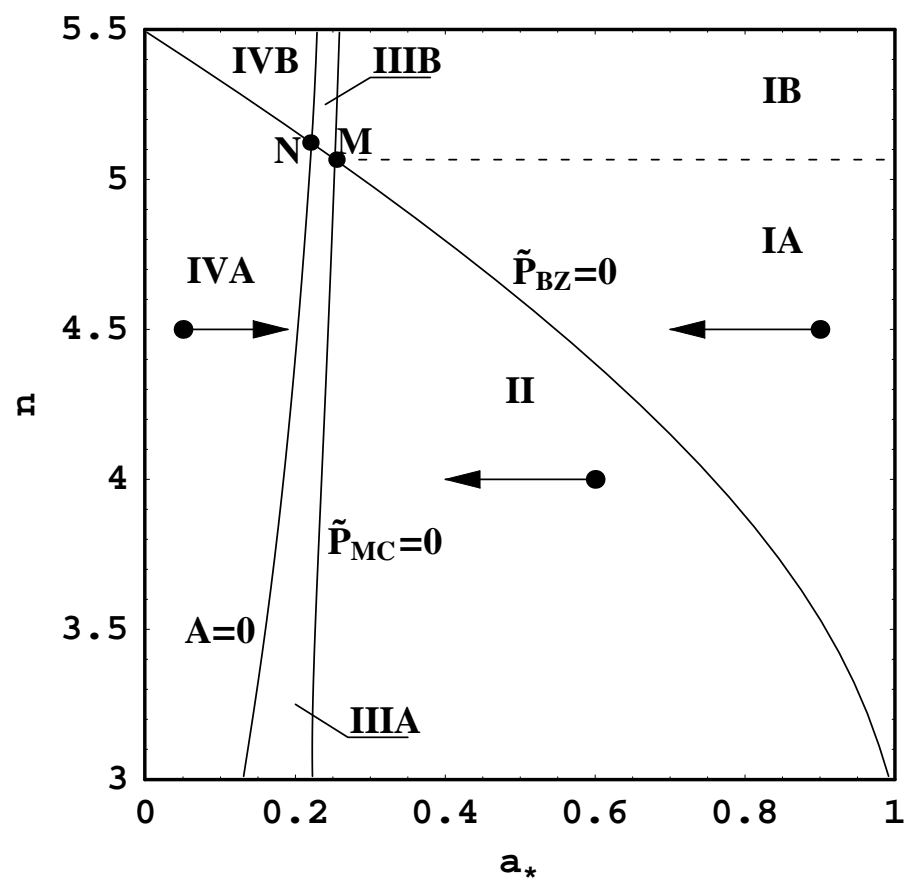

Fig. 5.- Parameter space of BH evolution in CEBZMC. 


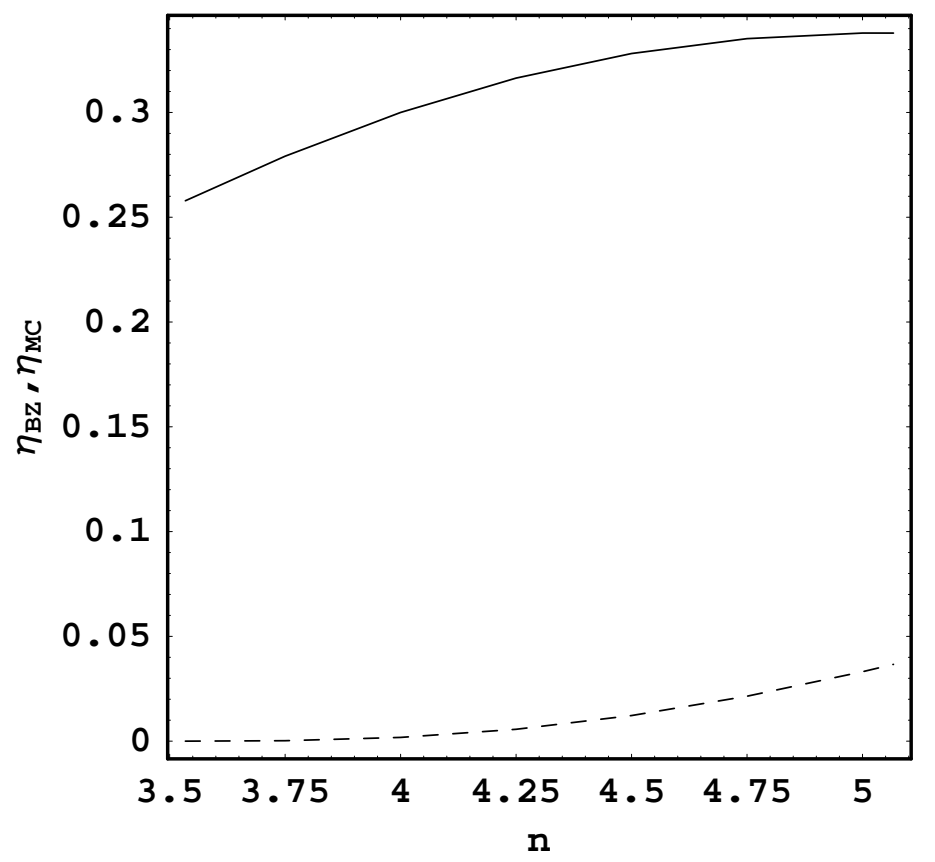

Fig. 6. - Curves of $\eta_{B Z}$ (dashed line) and $\eta_{M C}$ (solid line) vs. $n$ for $3.534<n<5.067$. 


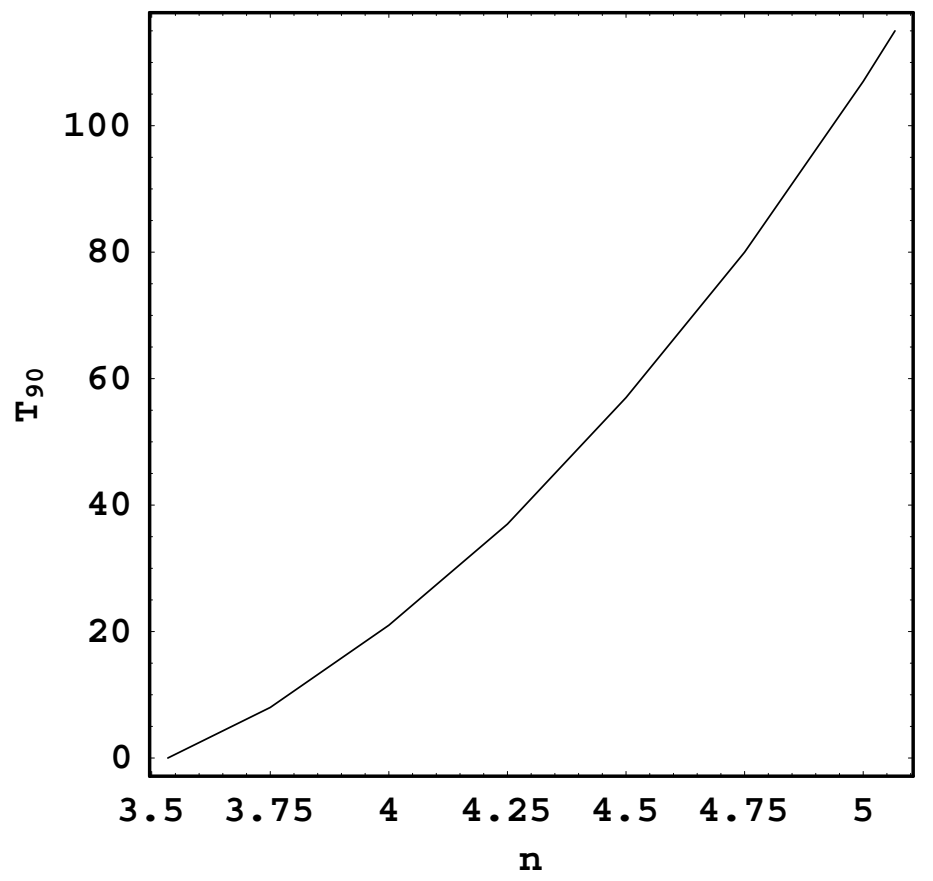

Fig. 7.- Curve of $T_{90}$ vs. $n$ for $3.534<n<5.067$. 